\title{
Multicenter Evaluation of Circulating Cell-Free DNA Extraction and Downstream Analyses for the Development of Standardized (Pre)analytical Work Flows
}

\author{
Rita Lampignano, ${ }^{1+}$ Martin H.D. Neumann, ${ }^{1+}$ Sabrina Weber, ${ }^{2,3}$ Vera Kloten, ${ }^{1}$ Andrei Herdean, ${ }^{4}$ \\ Thorsten Voss, ${ }^{5}$ Daniel Groelz, ${ }^{5}$ Anna Babayan, ${ }^{6}$ Marco Tibbesma, ${ }^{7}$ Martin Schlumpberger, ${ }^{8}$ \\ Francesca Chemi, ${ }^{9}$ Dominic G. Rothwell, ${ }^{9}$ Harriet Wikman, ${ }^{6}$ Jean-Pierre Galizzi, ${ }^{10}$ \\ Inger Riise Bergheim, ${ }^{11}$ Hege Russnes, ${ }^{11}$ Benedetta Mussolin, ${ }^{12}$ Serena Bonin, ${ }^{13}$ Christine Voigt, ${ }^{14}$ \\ Hanny Musa, ${ }^{15}$ Pamela Pinzani, ${ }^{16}$ Evi Lianidou, ${ }^{17}$ Ged Brady, ${ }^{9}$ Michael R. Speicher, ${ }^{2}$ Klaus Pantel, ${ }^{6}$ \\ Fay Betsou, ${ }^{18}$ Ed Schuuring, ${ }^{7}$ Mikael Kubista, ${ }^{4}$ Wim Ammerlaan, ${ }^{18}$ Markus Sprenger-Haussels, ${ }^{8}$
} Thomas Schlange, ${ }^{1 \neq}$ and Ellen Heitzer ${ }^{2,3^{*}}$ for the Innovative Medicines Initiative CANCER-ID Consortium

BACKGROUND: In cancer patients, circulating cell-free DNA (ccfDNA) can contain tumor-derived DNA (ctDNA), which enables noninvasive diagnosis, real-time monitoring, and treatment susceptibility testing. However, ctDNA fractions are highly variable, which challenges downstream applications. Therefore, established preanalytical work flows in combination with costefficient and reproducible reference materials for ccfDNA analyses are crucial for analytical validity and subsequently for clinical decision-making.

METHODS: We describe the efforts of the Innovative Medicines Initiative consortium CANCER-ID (http:// www.cancer-id.eu) for comparing different technologies for ccfDNA purification, quantification, and characterization in a multicenter setting. To this end, in-house generated mononucleosomal DNA (mnDNA) from lung cancer cell lines carrying known TP53 mutations was spiked in pools of plasma from healthy donors generated from 2 different blood collection tubes (BCTs). ccfDNA extraction was performed at 15 partner sites according to their respective routine practice. Downstream analysis of ccfDNA with respect to recovery, integrity, and mutation analysis was performed centralized at 4 different sites.
RESULTS: We demonstrate suitability of mnDNA as a surrogate for ccfDNA as a process quality control from nucleic acid extraction to mutation detection. Although automated extraction protocols and quantitative PCR-based quantification methods yielded the most consistent and precise results, some kits preferentially recovered spiked mnDNA over endogenous ccfDNA. Mutated TP53 fragments derived from mnDNA were consistently detected using both next-generation sequencing-based deep sequencing and droplet digital PCR independently of BCT.

CONCLUSIONS: This comprehensive multicenter comparison of ccfDNA preanalytical and analytical work flows is an important contribution to establishing evidence-based guidelines for clinically feasible (pre)analytical work flows. (C) 2019 American Association for Clinical Chemistry

Clinical utility of blood-based analytes as cancer biomarkers has been intensively investigated to overcome the limitations of conventional tissue biopsy sampling (e.g., invasiveness, accessibility, and heterogeneity). Cellfree circulating tumor DNA (ctDNA) ${ }^{19}$ has attracted increasing interest, as it reflects molecular characteristics of
${ }^{1}$ Bayer AG, Biomarker Research, Wuppertal, Germany; ${ }^{2}$ Institute of Human Genetics, Diagnostic and Research Center for Molecular BioMedicine, Medical University of Graz, Graz, Austria; ${ }^{3}$ Christian Doppler Laboratory for Liquid Biopsies for Early Detection of Cancer, Graz, Austria; ${ }^{4}$ TATAA Biocenter Ab, Gothenburg, Sweden; ${ }^{5}$ PreAnalytiX GmbH, Hombrechtikon, Switzerland; ${ }^{6}$ University Medical Center Hamburg-Eppendorf, Hamburg, Germany; ${ }^{7}$ University of Groningen, University Medical Center of Groningen, Groningen, the Netherlands; ${ }^{8}$ OIAGEN GmbH, Hilden, Germany; ${ }^{9}$ CR-UK Manchester Institute, University of Manchester, Manchester, UK; ${ }^{10}$ Servier, Suresnes, France; ${ }^{11}$ Department of Cancer Genetics, Institute of Cancer Research, Oslo University Hospital, Oslo, Norway; ${ }^{12}$ Candiolo Cancer Institute, FPO IRCCS, Candiolo, Italy; ${ }^{13}$ University of Trieste, DSM-Cattinara Hospital, Trieste, Italy; ${ }^{14}$ Alacris Theranostics GmbH, Berlin, Germany; ${ }^{15}$ Boehringer-Ingelheim, Ingelheim am Rhein, Germany; ${ }^{16}$ University of Florence, Florence, Italy; ${ }_{i}^{17}$ University of Athens, Athens, Greece; ${ }^{18} \mathrm{In}$ tegrated BioBank of Luxembourg, Dudelange, Luxembourg.

\footnotetext{
* Address correspondence to this author at: Institute of Human Genetics, Diagnostic and Research Center for Molecular BioMedicine, Medical University Graz, Neue Stiftingtalstrasse 6, 8010 Graz, Austria. E-mail ellen.heitzer@medunigraz.at.

${ }^{\dagger}$ R. Lampignano and M.H.D. Neumann contributed equally.

₹ T. Schlange and E. Heitzer contributed equally.

Received April 30, 2019; accepted August 5, 2019 Previously published online at DOI: 10.1373/clinchem.2019.306837

(C) 2019 American Association for Clinical Chemistry

${ }^{19}$ Nonstandard abbreviations: ctDNA, circulating tumor DNA; ccfDNA, circulating cell-free DNA; BCT, blood collection tube; VAF, variant allele frequency; mnDNA, mononucleosomal DNA; CNA, circulating nucleic acid; qPCR, quantitative PCR; ddPCR, droplet dig ital PCR; NGS, next-generation sequencing; MNase, micrococcal nuclease.
} 
tumor tissues (1-4). The ease of access of ctDNA makes it a promising analyte to improve cancer detection. ctDNA analysis already supports therapy guidance and longitudinal disease monitoring (5). Owing to its mainly apoptotic origin, circulating cell-free (ccfDNA) is highly fragmented, which complicates downstream analyses (5). Moreover, because nonmalignant cells-mainly from the hematopoietic system-also release ccfDNA, the tumor fraction is highly variable and often represents $<1 \%$ (6).

Therefore, the integration of ctDNA analysis into clinical practice faces a series of challenges and requires standardization to guarantee reliable and robust results. This is particularly an issue for (pre)analytical work flows as shown by poor interlab concordance (3, 7-10). Studies to evaluate aspects influencing preanalytical sample handling have demonstrated that the choice of blood collection tubes (BCTs) and their associated stabilization reagents, storage temperature and duration, plasma preparation, and extraction protocols can significantly affect integrity, purity, and yield of ccfDNA (11-15). These confounding factors can lead to considerable variability in ccfDNA extraction efficacy, quantification, and molecular characterization (16-25).

The Innovative Medicines Initiative (IMI) program CANCER-ID is a public-private partnership active in standardizing liquid biopsy assays. Key deliverables of the consortium are the evaluation of technologies and best practice recommendations for blood-based biomarker analysis to establish criteria for benchmarking different technologies.

Here, we describe efforts of the consortium in comparing different technologies for ccfDNA purification, quantification, and characterization to establish standardized (pre)analytical work flows for ccfDNA analysis. To create a meaningful model for an external quality assessment of DNA extraction, quantification, and mutation analysis at various variant allele frequencies (VAFs), we evaluated the use of mononucleosomal DNA (mnDNA), which potentially mimics naturally occurring ccfDNA more closely than standards based on sheared genomic DNA as an in-process control.

\section{Materials and Methods}

We compared 7 different extraction kits in a multicenter setting and centrally evaluated various work flows for cfDNA quantification and mutation detection using orthogonal methods (Fig. 1). To assess various levels of mutant fragments, we used 2 spike sets of mnDNA derived from 2 cancer cell lines. A detailed description of the methods can be found in material included in the Data Supplement that accompanies the online version of this article.

\section{PREPARATION OF SPIKED-IN PLASMA POOLS}

To mirror the nucleosome-protected nature of ccfDNA, we generated mnDNA from 2 non-small cell lung cancer cell lines (NCl-H441 and NCI-H1573, both purchased from ATCC) as a reference material. Both cell lines carry a homozygous TP53 ${ }^{20}$ mutation (NM_000546.4: c.473G $>$ T p.Arg158Leu for NCl-H441 and NM_ 000546.4: c.743G $>$ T p.Arg248Leu for NCl-H1573; hereinafter referred to as R158L and R248L), which was used as readout for downstream analysis (26). Nucleosomes were prepared using the Nucleosome Preparation Kit (Active Motif). After quality control on a Tape Station (Agilent Technologies), 2 different mixed mnDNA preparations, resulting in different VAFs of the TP53 mutations (see Table 1 in the online Data Supplement), were spiked into 2 different plasma pools derived from healthy donors. To assess the feasibility of work flows with cfDNA extracted from the most commonly used BCTs in the field, blood samples from 15 and 28 healthy donors were drawn into Cell-Free DNA BCT® (Streck) and PAXgene Blood ccfDNA Tube (PreAnalytiX), respectively. Spiked plasma pools were aliquoted to 4.25 $\mathrm{mL}$ and shipped to each participating laboratory $(\mathrm{n}=$ 15). For each BCT, each site received 3 replicates of spike set I and 2 replicates with spike set II (see Table 1 in the online Data Supplement).

\section{CCFDNA EXTRACTION}

Plasma samples were thawed on ice; ccfDNA extraction was performed according to the manufacturer's protocols. ccfDNA extraction was performed using 6 different extraction kits, including the QIAamp Circulating $\mathrm{Nu}$ cleic Acid (CNA) kit (4 sites), QIAsymphony circulating DNA kit (4 sites), QIAamp MinElute ccfDNA kit (4 sites) (all QIAGEN), Maxwell RSC ccfDNA Plasma Kit AX1115 (3 sites), Maxwell RSC ccfDNA Plasma Kit AS1480 (Promega) (1 site), and Chemagic CNA 4k kit special (PerkinElmer) (1 site) following the manufacturer instructions without any deviations (see Table 2 in the online Data Supplement). Eluates were aliquoted and shipped on dry ice to Bayer AG, QIAGEN, Medical University of Graz, and TATAA Biocenter for centralized downstream analyses (Fig. 1).

\section{cCFDNA QUANTIFICATION}

ccfDNA was centrally quantified at Bayer using the Qubit High Sensitivity kit (Thermo Fisher) and at QIAGEN using the quantitative PCR (qPCR)-based Quantiplex Pro assay (QIAGEN). Moreover, we used droplet digital PCR (ddPCR; Bio-Rad) data of 2 TP53 assays (R158L and R248L; both Bio-Rad) for absolute

\footnotetext{
${ }^{20}$ Human Gene: TP53, tumor protein p53.
} 


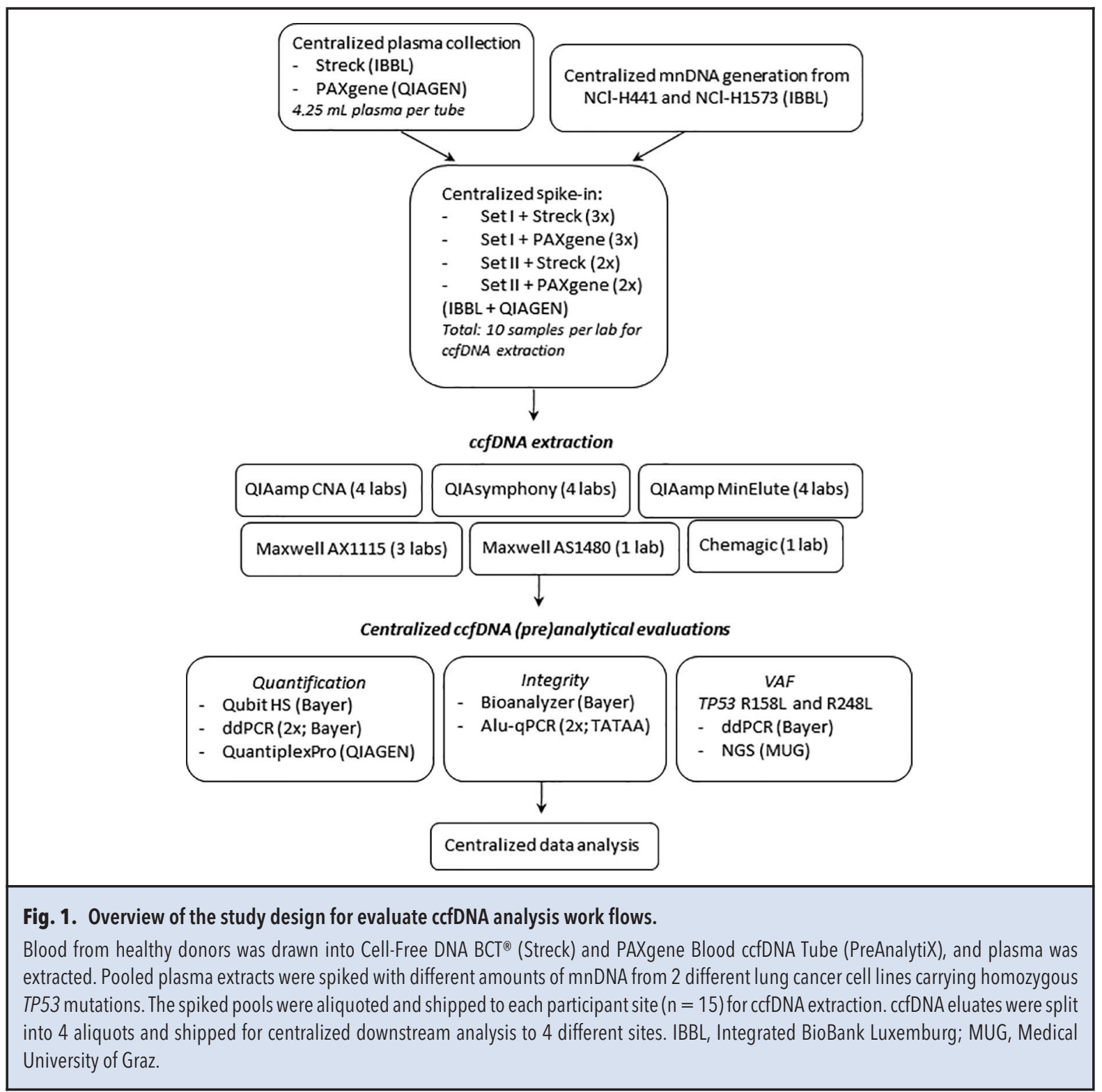

quantification purposes and combined positive mutant and wild-type droplets to calculate the overall recovery of mutated mnDNA and intrinsic ccfDNA from the donors.

\section{ASSESSMENT OF ccfDNA FRAGMENT LENGTH AND INTEGRITY}

Fragment sizes were evaluated at Bayer AG on a Bioanalyzer (Agilent Technologies) using the High Sensitivity DNA kit according to the manufacturer's protocol. DNA integrity was assessed by measuring 2 different AluI PCR products with lengths of $60 \mathrm{bp}$ and $187 \mathrm{bp}$ at the TATAA Biocenter and calculating the ratio between their quantitation cycles values (Alu-187/Alu-60).
MUTATION ANALYSIS OF TP53 R158L AND R248L

TP53 mutations were analyzed using ddPCR and deep sequencing. For each TP53 mutation, a specific ddPCR assay was performed using the Bio-Rad QX200 ${ }^{\mathrm{TM}}$ platform including no template controls $(\mathrm{n}=8)$. Data were analyzed using the QuantaSoft ${ }^{\mathrm{TM}}$ analytical software version 1.7.4 (Bio-Rad). Fluorescent signals of negative controls were recorded as background to establish cutoff levels. Results were expressed as both number of mutants/ ccfDNA eluate (in microliters) as well as VAF (ratio between number of mutant and wild-type droplets after correction using the Poisson distribution). The limit of detection of the ddPCR assays was $0.1 \%$. 
For next-generation sequencing (NGS), targetspecific primers spanning the TP53 mutations were designed and tagged with Illumina-specific adapter sequences (see Table 3 in the online Data Supplement). Amplicon sizes were $120 \mathrm{bp}$ and $113 \mathrm{bp}$ for the R158L and R248L mutations, respectively. Deep sequencing was performed as previously described (27). Paired-end sequencing was performed on MiSeq and NextSeq sequencers (both Illumina), and reads were analyzed as previously described $(27,28)$. VAF was calculated as the number of mutated reads divided by number of wildtype reads. As we did not use a molecular barcoding strategy, this deep NGS assay can reliably detect a VAF of $1 \%$.

\section{STATISTICAL ANALYSIS}

A linear mixed-effects model was used to assess intralab variability. Comparisons between different extraction methods and downstream approaches were performed via the 2-way ANOVA multiple comparison test. Twotailed tests with $P<0.05$ were considered statistically significant. Data analysis and visualization was performed using Prism 7.0 (GraphPad Software) and R (version 3.5.2) as well as R Studio software (R Studio). Data revealed by the Bioanalyzer and linear mixed-effects models were analyzed using the statistical programming language $\mathrm{R}$ (29). Because different donors were used for the PAXgene ccfDNA and Streck BCT plasma pools, we did not perform a statistical comparison between the tubes.

\section{Results}

\section{PREPARATION OF mnDNA AND VALIDATION OF SPIKED} PLASMA SAMPLES

Micrococcal nuclease (MNase) treatment resulted in a sample volume of $350 \mu \mathrm{L}$. DNA isolation from $50 \mu \mathrm{L}$ of MNase preparation yielded 143 and $89 \mathrm{ng} / \mu \mathrm{L}$ in $50 \mu \mathrm{L}$ of elution buffer of purified H441 and H1573 mnDNA fragments, respectively. Analysis on a 4200 TapeStation revealed $95 \%$ and $89 \%$ of purified $\mathrm{H} 441$ and $\mathrm{H} 1573$ mnDNA fragments in the range of 50 to $700 \mathrm{bp}$, peaking at $149 \mathrm{bp}$ and $142 \mathrm{bp}$, respectively (data not shown). To assess a wide spectrum of VAFs, 2 different mixes of MNase-treated cell line DNA carrying homozygous TP53 mutations were spiked into pools obtained from a total of 43 healthy donors (Streck $\mathrm{n}=15$; PAXgene $\mathrm{n}=$ 28) (see Table 1 in the online Data Supplement). Intrinsic ccfDNA concentrations of plasma pools were 17.06 and $21.3 \mathrm{ng} / \mathrm{mL}$ plasma for Streck and PAXgene ccfDNA, respectively. Size distribution analysis revealed the presence of 2 predominant fragment sizes in the range of $140 \mathrm{bp}$ and $166 \mathrm{bp}$, corresponding to the spiked-in mnDNA and donor-derived ccfDNA, respectively (see Fig. 1 in the online Data Supplement).

\section{ASSESSMENT OF INTRALABORATORY VARIABILITY}

Although a well-powered statistical analysis of intralab variability was not applicable to the limited number of replicates used in this study, we assessed intralaboratory variation using a linear mixed-effects model. Intralab variabilities of Qubit measurements ranged around 10\% with only a few laboratories as outliers (Fig. 2 here and Figs. 2 and 3 in the online Data Supplement). Therefore, all subsequent statistical comparisons reported here refer to the ANOVA tests only.

\section{QUANTITATIVE COMPARISON OF DIFFERENT cCFDNA EXTRACTION METHODS}

Quantification of ccfDNA was performed using Qubit, qPCR, and the 2 TP53 ddPCR assays (see Table 4 in the online Data Supplement). A common observation was that measurements from extraction of the same laboratory were closer to each other than between laboratories (see Fig. 2 here and Fig. 2 in the online Data Supplement). The Qubit method demonstrated the greatest variability, with the QIAamp CNA kit presenting the widest range of yields for both BCTs (see Fig. 2 here and Fig. 2 and Tables 5 and 6 in the online Data Supplement). The 2 ddPCR assays and the Quantiplex Pro assays showed a lower degree of variability and good overall concordance. The Maxwell AX1115 revealed the lowest variation but was accompanied by lower yields of recovered ccfDNA measured with PCR-based methods compared with the QIAGEN kits (for $P$ values, see Table 5 in the online Data Supplement) (see Fig. 2 here and Fig. 2 in the online Data Supplement). In contrast, Qubit quantification of ccfDNA from Maxwell AX1115 extraction indicated significantly higher ccfDNA recovery from Streck plasma (see Fig. 2A here and Fig. 2A in the online Data Supplement) compared with the other quantification methods, and this same trend was observed for plasma derived from PAXgene ccfDNA tubes (see Fig. 2B here and Fig. 2B in the online Data Supplement). As extractions with Maxwell AS1480 and the Chemagic kit were performed only at a single site, these results were not compared with a multisite usage. Nevertheless, whereas yields of the Maxwell AS1480 were similar to the AX1115 kit, the Chemagic kit recovered the lowest yields of ccfDNA. Notably, measurements recorded for the spike set II, in which a lower amount of mnDNA was spiked in and only 2 replicates were used, exhibited on average higher variability in recovery (see Fig. 2 and Table 6 in the online Data Supplement). Although not directly comparable owing to different sets of donor plasmas, both BCTs showed similar results.

\section{ccFDNA FRAGMENT SIZE DISTRIBUTION AND INTEGRITY} ASSESSMENT

Based on the validation of the spiked plasma pools, we expected 2 major peaks after Bioanalyzer profiling, i.e., at 


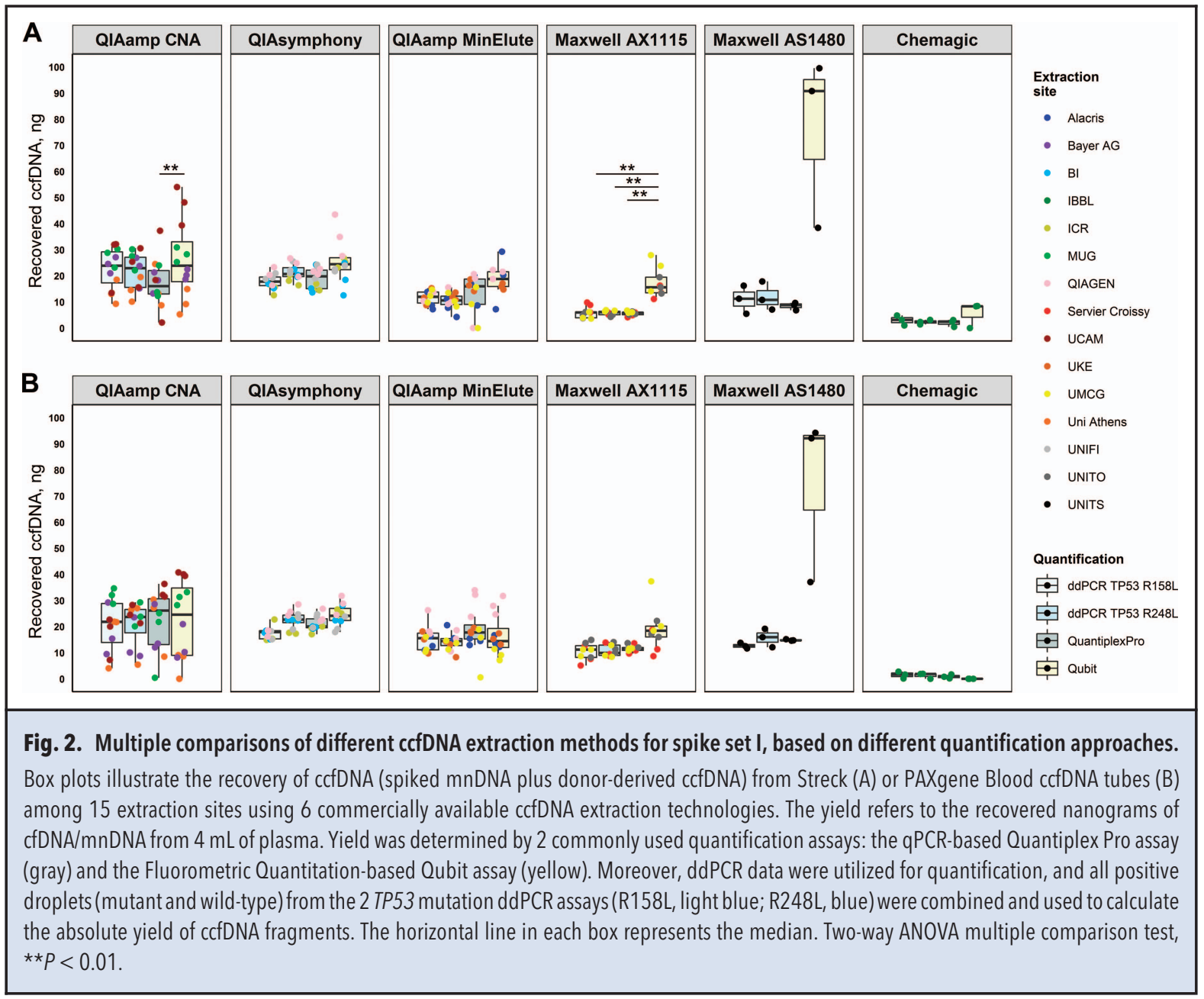

140 bp for spiked mnDNA and 166 bp for intrinsic ccfDNA (see Fig. 1 in the online Data Supplement). Both peaks could be observed in all eluates but most prominently for the QIAGEN kits (see Fig. 3 here and Fig. 4 in the online Data Supplement). This can be explained by the fact that $1 \mu \mathrm{L}$ of each eluate was applied to the chip rather than a normalized amount of ccfDNA. Therefore, the size distribution is more evident at higher concentrations (QIAsymphony and QIAamp CNA). Likewise, fragments in the range of $320 \mathrm{bp}$ and $480 \mathrm{bp}$, corresponding to dinucleosomal and trinucleosomal DNA, were observed only when at least $0.4 \mathrm{ng}$ of ccfDNA was applied to the chip. However, no presence of high molecular weight DNA was observed for any of the kits, indicating that both BCTs effectively protect from release of high molecular weight DNA from (blood) cells.

The integrity of ccfDNA was measured as the ratio between 2 ALU1 assays targeting DNA fragments of different lengths of $187 \mathrm{bp}$ and $60 \mathrm{bp}$ (Alu-187 and Alu60). Whereas all QIAGEN kits showed similar integrity indices in the range of 1.30 to 1.46 with a median of 1.39, fragments extracted with the Maxwell AX1115 kits showed significantly lower integrity indices, ranging from 1.34 to 1.35 with a median of 1.34 , irrespective of spike set or BCT (Fig. 4; for $P$ values, see Table 7 in the online Data Supplement). Integrity indices for Maxwell AS1480 and Chemagic were slightly lower than for the QIAGEN kits but higher than for the Maxwell AX1115.

\section{DETECTION OF TP53 MUTATIONS R158L AND R248L}

To assess the recovery and allele frequency of the spiked mutant mnDNA, all eluates were analyzed with 2 orthogonal methods, mutation-specific ddPCR and deep sequencing (NGS) TP53 assays, respectively (see Table 8 in the online Data Supplement). Similar to the recovery rates, VAFs established from extraction from single laboratories were highly concordant, whereas VAFs between laboratories showed higher variability (see Fig. 5 here and Fig. 5 in the online Data Supplement). Nevertheless, VAFs calculated from ddPCR and NGS from each site were strongly correlated by linear regression with an av- 

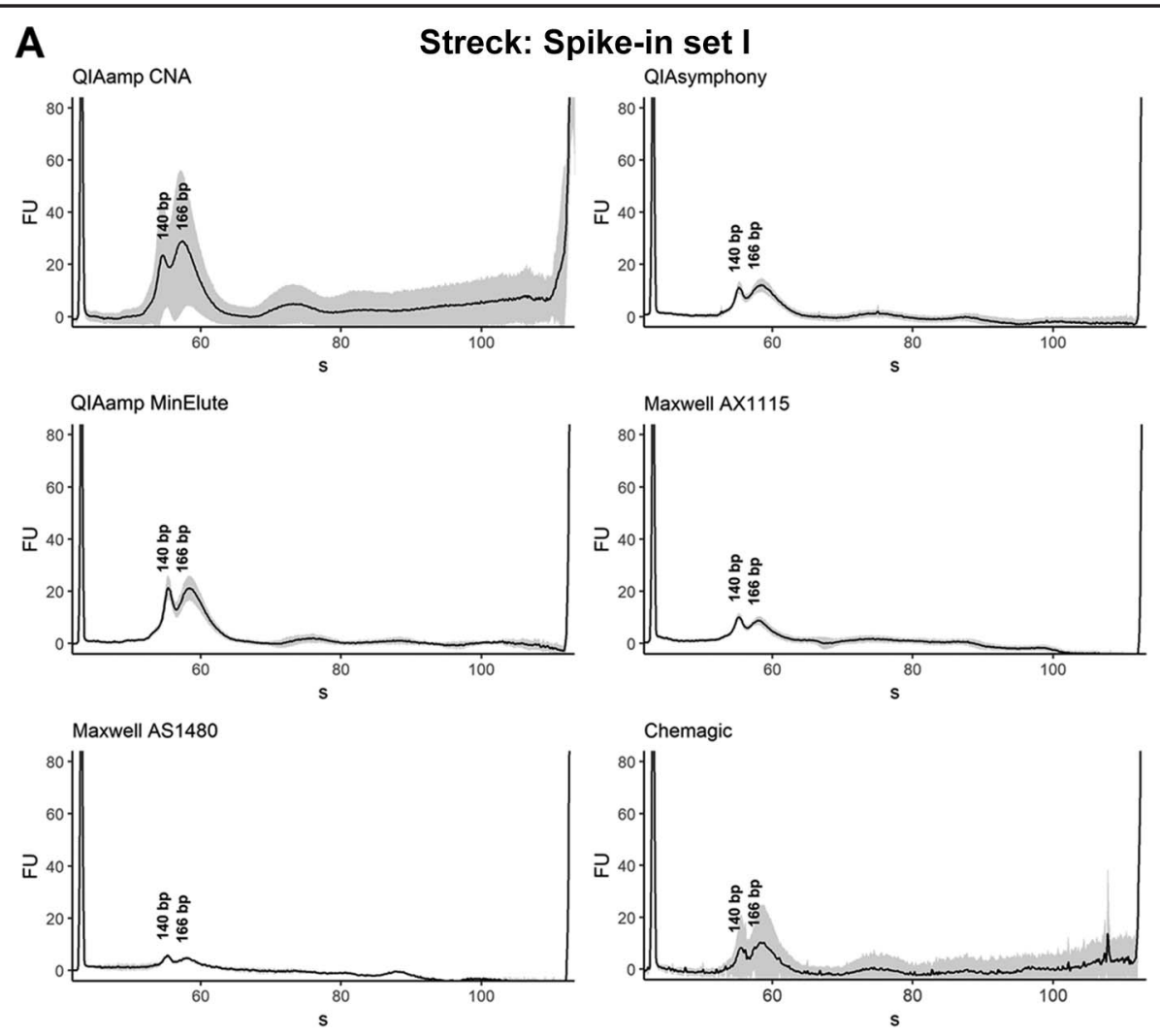

Fig. 3. Multiple comparisons of different ccfDNA extraction methods for spike-in set I, based on size of ccfDNA fragments.

Bioanalyzer electropherograms illustrate profiles of the fragments of mnDNA and intrinsic ccfDNA extracted from Streck (A) and PAXgene (B) tubes by 6 different commercially available ccfDNA extraction technologies. For all approaches, 2 major peaks of $140 \mathrm{bp}$ and of $166 \mathrm{bp}$ were detected, respectively. Dinucleosomes and trinucleosomes could be observed for QIAamp CNA and QIAsymphony only. The SD is represented in gray. FU, fluorescent units; $s$, seconds (aligned migration time).

Continued on page 155

erage Lin concordance coefficient of 0.9264 (range, 0.7979-0.9854), indicating a good concordance between the methods (see Fig. 6 in the online Data Supplement).

Considering all measurements, for the QIAGEN kits, both mutation detection approaches exhibited a good concordance with respect to VAF, irrespective of spike set or BCT (see Fig. 5 here and Fig. 5 and Tables 9 and 10 in the online Data Supplement). In contrast, the Maxwell AX1115 showed significantly higher VAFs with a much broader range when using ddPCR compared with NGS (see Fig. 5, A and B, here and Fig. 5, A and B, and Tables 9 and 10 in the online Data Supplement) (for $P$ values, see Tables 9 and 10 in the online Data Supplement). For example, for spike set I (PAXgene), VAFs ranged from $29 \%$ to $81 \%$ and from $0.7 \%$ to $9 \%$ for R158L and R248L, respectively, in contrast with 29\% to $42 \%$ and $2 \%$ to $7 \%$ obtained from the QIAsymphony extractions.
Moreover, VAFs obtained from Maxwell AX1115 eluates were slightly higher than those from the QIAGEN kits, with VAFs for ddPCR being even higher. Similar results were observed from Maxwell AS1480, although these results were obtained from a single site only. This observation is in line with a significantly different recovery efficiency of mutant mnDNA and donor-derived ccfDNA fragments (see Fig. 5C here and Fig. 5C and Tables 9 and 10 in the online Data Supplement). The Maxwell kits, in particular the Maxwell AX1115, showed substantially lower recovery of the slightly larger wild-type ccfDNA fragments from the donors, pointing toward more efficient recovery of the spiked mnDNA. The Chemagic kit exhibited the lowest recovery of mnDNA.

\section{Discussion}

Although the potential role of ctDNA as a blood-based cancer biomarker has been highlighted by numerous 

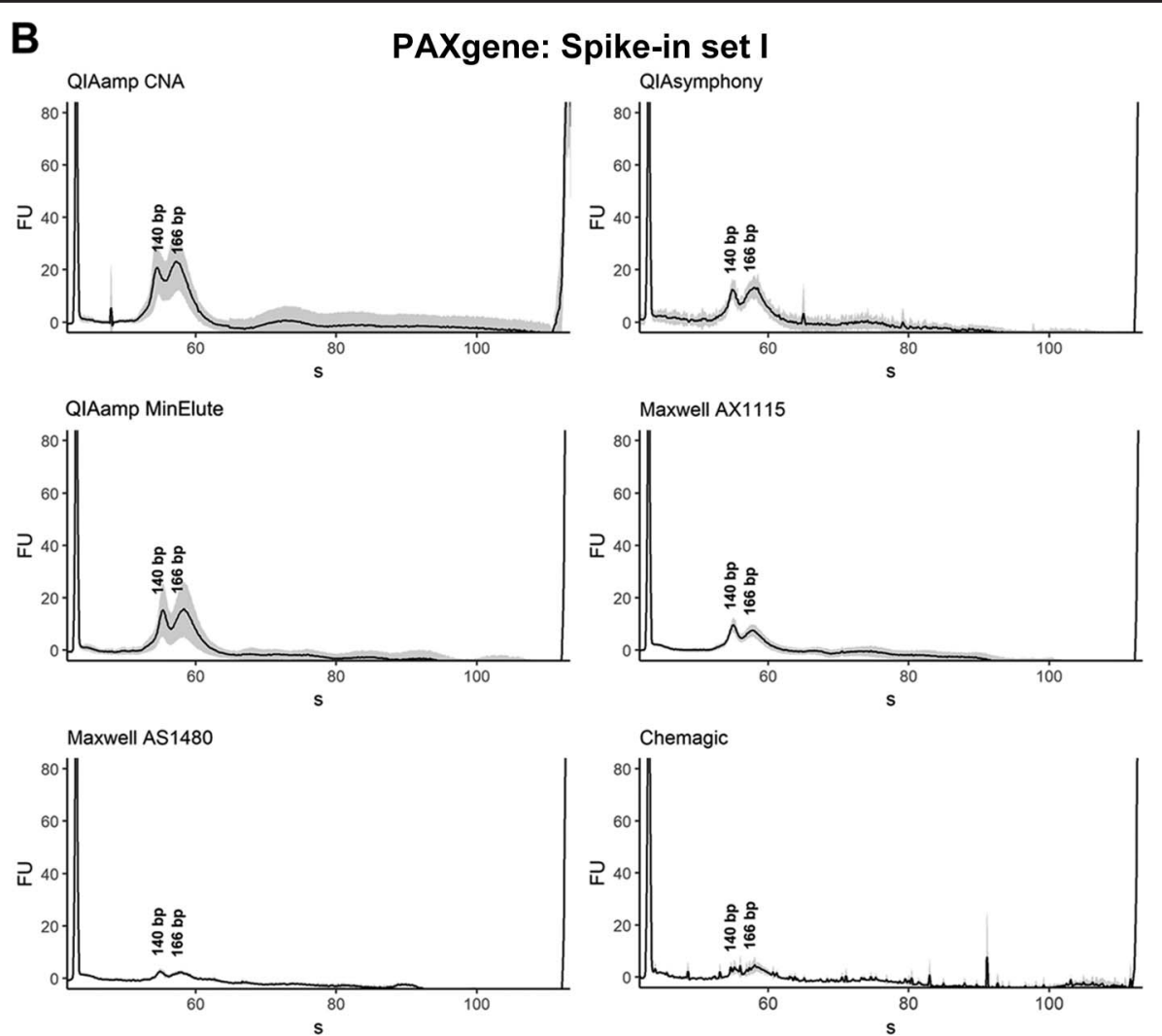

Fig. 3. Continued.

groups and 2 assays for ctDNA analysis have been approved by the Food and Drug Administration as part of companion diagnostics in colon and lung cancer $(1,3,4,6,30,31)$, standardized (pre)analytical work flows are still an unmet need for the broad integration of ctDNA-based applications into the clinic. Despite existing efforts of comparing ccfDNA extraction technologies and assessing confounding factors such as storage temperature or duration $(16-25,32-34)$, further systematic investigations will be required to reach a consensus for standard operating procedures and best practice guidelines. To this end, CANCER-ID collaborates with the SPIDIA4P program (http://www.spidia.eu) that is developing CEN (European Committee for Standardization) technical preanalytical standards, including those for ccfDNA (CEN/TC 16835-3, https://standards.cen.eu/).

Here we present a multicenter comparison of ccfDNA extraction and quantification using multiple preanalytical and analytical work flows. We report for the first time the use of mnDNA spiked into plasma from healthy individuals collected in Streck and PAXgene ccfDNA BCTs as a process quality control from nucleic acid extraction to mutation detection. mnDNA closely mirrors the nucleosome-bound nature of ccfDNA and better mimics naturally occurring ccfDNA fragment properties in terms of size distribution, blunt fragment ends, and nucleosome patterns than sonicated cell line DNA or synthetic oligonucleotides. Recently, the utility of mnDNA for test performance assessment for $\mathrm{ctDNA}$ somatic mutation testing was demonstrated in a multicenter study using a targeted capture NGS approach (35). The authors demonstrated an almost identical performance of mnDNA compared with ccfDNA samples from tumor patients. However, 1 limitation was that the ccfDNA extraction process was not evaluated in the study. Using mnDNA, we assessed the performance of 6 commercially available ccfDNA extraction methods, including the column-based QIAamp CNA and QIAamp MinElute ccfDNA kits and the automated beads-based QIAsymphony, Maxwell AX1115 and AS1480, and Chemagic protocols, all routinely used by $\geq 1$ partners of the CANCER-ID consortium in combination with orthogonal downstream mutation analysis approaches.

In general, for all analyzed parameters, the measured values were more consistent within laboratories than between laboratories. Regarding quantification, the Qubit assay produced slightly higher values with a much broader range than the PCR-based quantitative tech- 


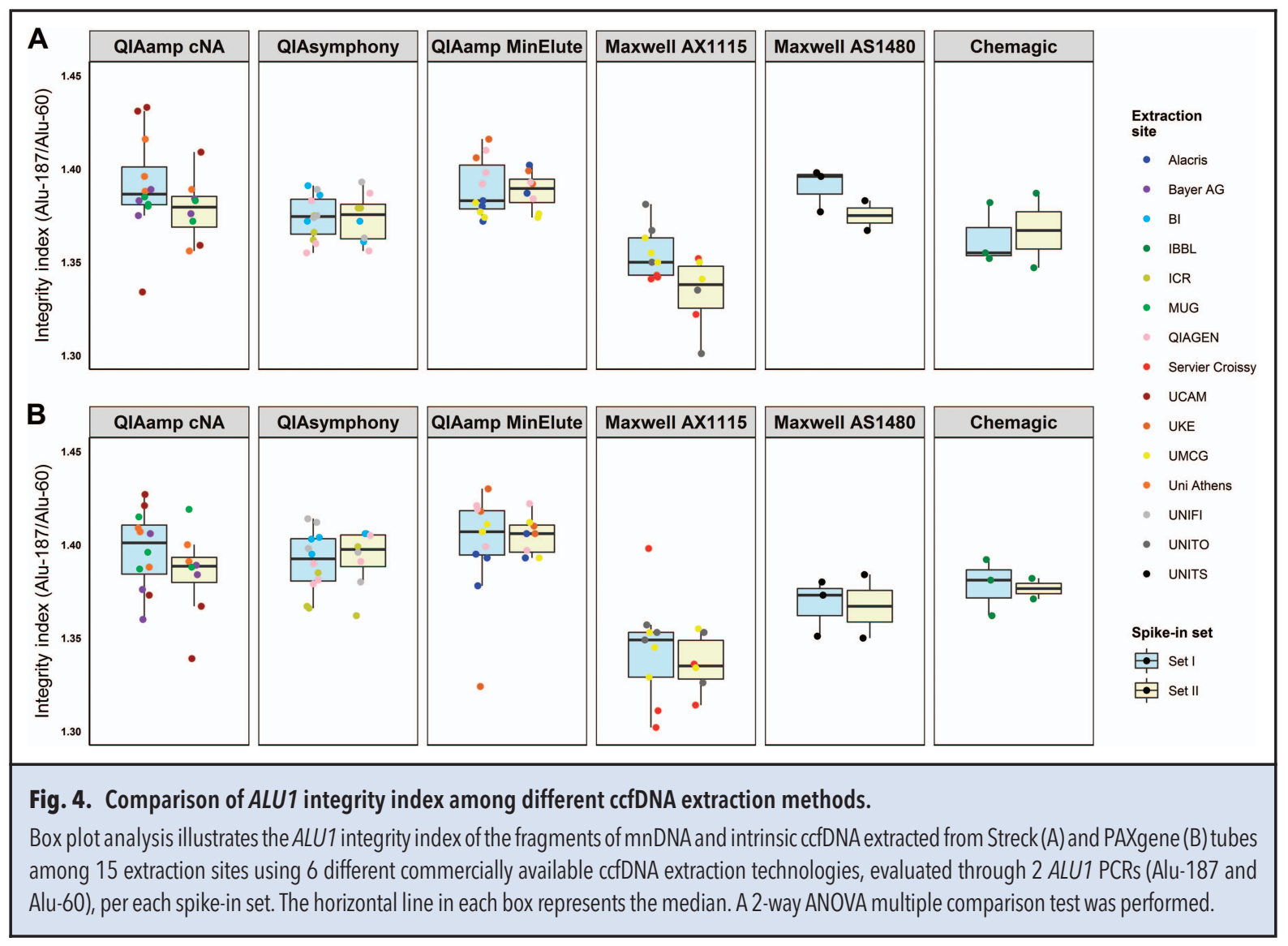

niques, possibly because of overestimation by this method, especially at the limit of quantification (21). In contrast, yields established from GPCR and mutationspecific ddPCR were highly consistent. Therefore, we recommend using PCR-based assays when an accurate measure of ccfDNA quantity is required for downstream analysis. Moreover, the automated QIAsymphony protocol showed the best concordance for all quantification methods among participating sites, followed by the also automated Maxwell AX1115 platform, irrespective of the BCT used or of the spike set. It is of note that because of the lower overall amount of spiked mnDNA and the use of only 2 replicates, the variability for spike set II was generally higher. The QIAamp CNA showed the highest recovery, independent of the spike set or the BCT. These data are consistent with previous reports, which consider the QIAamp CNA kit as the gold standard approach $(16,17,20-25,33)$. Nevertheless, our observations imply the importance of integrating automated approaches into clinical routine practice to reduce operator-based variability and, in doing so, increasing the concordance of results among different sites.

Recent studies suggest that fragment sizes of tumorderived fragments might differ from normal ccfDNA
(36-39). As the recovery of long/short ccfDNA fragments is of high interest and may affect the detection of rare cancer-related mutations, we additionally assessed the length distribution of the recovered fragments. Bioanalyzer profiling revealed 2 peaks in the range of approximately $140 \mathrm{bp}$ and of approximately $166 \mathrm{bp}$ in all eluates, thus corresponding to the in-house generated mnDNA spiked-in control and the donor-derived ccfDNA, respectively, which aligns with previous observations (36-38). Predominant peaks were particularly detected for methods that yielded higher ccfDNA concentrations in the eluates (QIAsymphony and QIAamp MinElute kits), irrespective of the spike set and BCT. An enrichment of high molecular weight DNA fragments ( $\geq 1000$ bp), which could indicate genomic DNA released by nucleated blood cells, was not observed (40).

A PCR-based DNA integrity approach revealed similar integrity indices for all QIAGEN kits, whereas the automated Maxwell AX1115 protocol showed significantly lower values, suggesting that this protocol preferentially isolates longer donor-derived ccfDNA over the smaller spiked-in mnDNA. This was not in concordance with ddPCR data, in which, compared with the QIAGEN kits, an equal proportion of mutated mnDNA frag- 


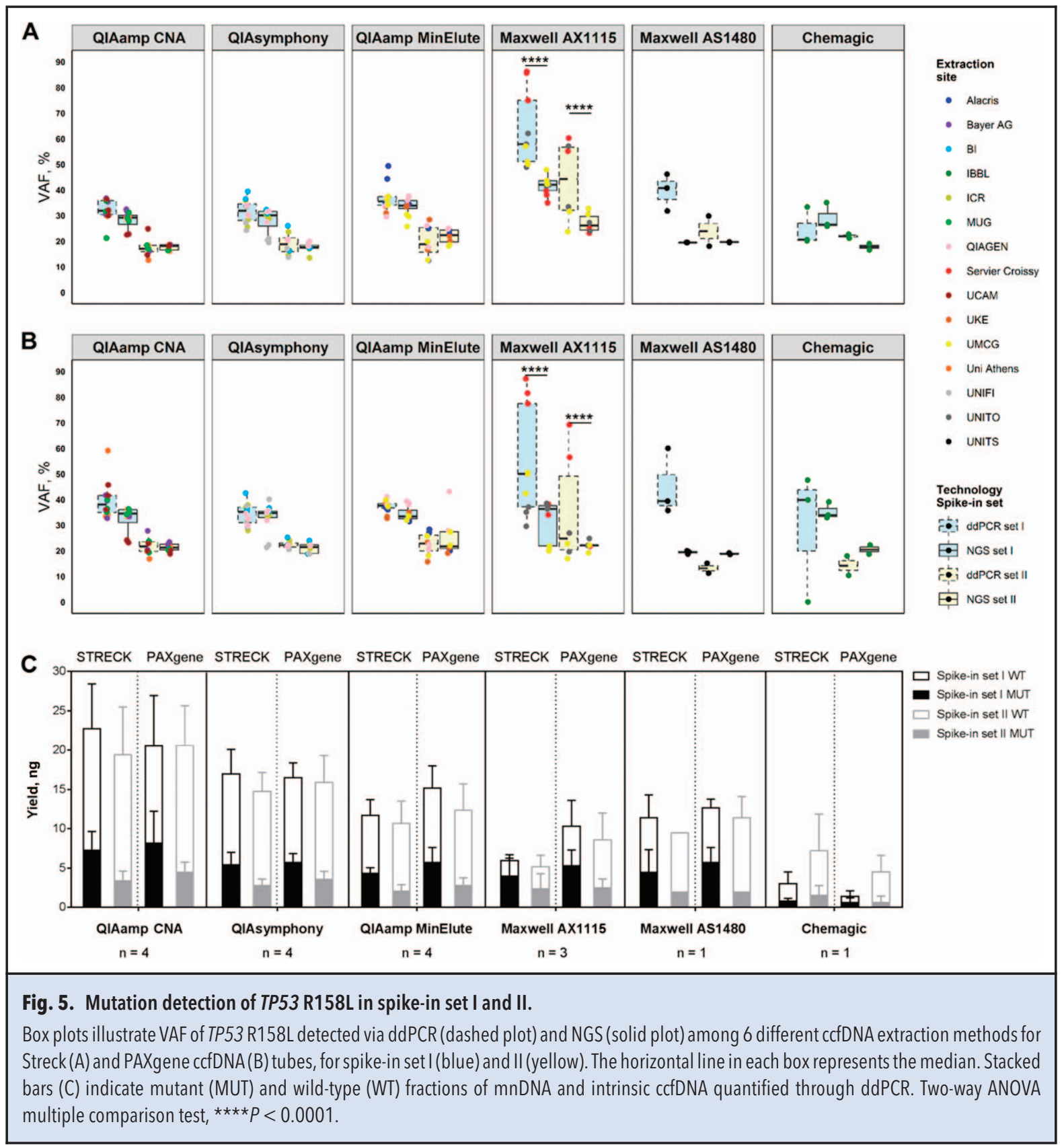

ments ( $140 \mathrm{bp}$ ) but a smaller proportion of intrinsic wildtype ccfDNA fragments (166 bp) was observed (see Fig. 5 here and Fig. 4 in the online Data Supplement). Therefore, despite a lower overall yield of recovered fragments, the Maxwell AX1115 showed an artificially increased VAF of TP53 mutations in Streck tubes and a similar trend in PAXgene ccfDNA tubes. In this context, amplicon size should also be taken into account for ctDNA assay design. In our study, the ddPCR amplicons were of $90 \mathrm{bp}$ in length in contrast with 113 and $120 \mathrm{bp}$ of the NGS assays, potentially leading to a preferential amplifi- cation of the shorter mnDNA. This might explain the slightly higher - albeit for the majority of eluates, not significantly different-VAFs observed with ddPCR. Only eluates from the Maxwell AX1115 kit showed significantly higher VAFs for both TP53 mutations compared with NGS, but this was more prominent in the samples with higher input (spike set I).

NGS seemed to present more consistent results than ddPCR. This observation can be explained by the fact that NGS used a 4-fold higher input amount compared with ddPCR. Therefore, it is likely that stochastic varia- 
Table 1. Important findings and recommendations for ctDNA work flows.

\begin{tabular}{|c|c|}
\hline \multirow[t]{2}{*}{ Extraction } & $\begin{array}{l}\text { Automated approaches should be integrated into the clinical routine to reduce operator-based } \\
\text { variability and hands-on time. }\end{array}$ \\
\hline & Various extraction kits differ in their efficiency of recovering different fragment lengths. \\
\hline \multirow[t]{2}{*}{ Quantification } & $\mathrm{qPCR}$ is more accurate and precise than Qubit. \\
\hline & $\begin{array}{l}\text { qPCR should be used in cases when only a little material is available and accurate quantification } \\
\text { is required. }\end{array}$ \\
\hline \multirow[t]{2}{*}{ Downstream analysis } & $\begin{array}{l}\text { Deep sequencing and ddPCR methods can be effectively utilized in a clinical setting in } \\
\text { combination with a variety of extraction kits and BCTs. }\end{array}$ \\
\hline & $\begin{array}{l}\text { The amount of input DNA should be taken into account for assay validation, as it contributes to } \\
\text { the variability of the downstream analysis. }\end{array}$ \\
\hline Process control & $\begin{array}{l}\text { mnDNA closely mirrors the nucleosome-bound nature of ccfDNA and can be used as a process } \\
\text { quality control from nucleic acid extraction to mutation detection. }\end{array}$ \\
\hline Assay design & Fragment length and amplicon size should also be taken into account for ctDNA assay design. \\
\hline \multirow[t]{2}{*}{ Validation } & $\begin{array}{l}\text { A continuous use of the same method based on validated standardization of operating } \\
\text { procedures should be pursued. }\end{array}$ \\
\hline & $\begin{array}{l}\text { Because of unavoidable biases to DNA integrity and mutation detection rate of various work } \\
\text { flows, some form of verification is required when changing parts of the work flow to } \\
\text { determine whether the new work flow complies with the previous assertions. }\end{array}$ \\
\hline
\end{tabular}

tion in pipetting in combination with lower numbers of target molecules contributed to the variability of ddPCR results. Yet, a good concordance was achieved between VAFs obtained from NGS and ddPCR within laboratories. It is of note that the major aim of this study was the assessment of different work flows, including orthogonal methods for mutation detection in combination with MNase-treated DNA rather than testing the analytical sensitivity and reproducibility of ddPCR and NGS. To this end, we decided to spike levels of mutated DNA that can be reliably detected with both methods, ranging from $2.5 \%$ to $30 \%$. When it comes to the detection of rare variants (VAF $<1 \%$ ), ddPCR may outperform conventional NGS unless molecular barcoding strategies are used (6). Nevertheless, our data suggest that both methods can be effectively used in a clinical setting in combination with the tested extraction kits and BCTs. However, a limitation of our study was that owing to 2 different donor sets for the 2 $\mathrm{BCTs}$, a direct comparison of them was not possible. Furthermore, it is important to add that when orthogonal methods are used for mutation detection, confounding factors such as input amounts or amplicon sizes may influence the results and add to variability.

Because the extraction method and the downstream application might introduce unavoidable biases to DNA integrity and the mutation detection rate, we highly recommend the continuous use of the same method based on validated standard operating procedures to obtain comparable results. If a laboratory is considering changing parts of the work flow, some form of verification is required to determine whether the new work flow complies with the previous assertions. For this purpose and for comprehensive external quality assessments, mixtures of mnDNAs from mutant and wild-type cell lines with defined VAFs spiked into synthetic plasma may present an inexhaustible quality measure for various methods.

In summary, we report findings highly relevant for the development of standard operating procedures and validation of clinically relevant tests (Table 1). Our data are a step toward standardization of preanalytical processes and the verification and validation of ctDNA assays, which is essential in proving their clinical utility, and finally enabling widespread use of ctDNA in clinical routine practice.

Author Contributions: All authors confirmed they have contributed to the intellectual content of this paper and have met the following 4 requirements: (a) significant contributions to the conception and design, acquisition of data, or analysis and interpretation of data; (b) drafting or revising the article for intellectual content; (c) final approval of the published article; and (d) agreement to be accountable for all aspects of the article thus ensuring that questions related to the accuracy or integrity of any part of the article are appropriately investigated and resolved.

R. Lampignano, concept and study design, data analysis, statistical analysis, conduction of experiments, manuscript preparation; M.H.D. Neumann, concept and study design, statistical analysis, conduction of experiments, manuscript preparation; S. Weber, conduction of experiments, data analysis; V. Kloten, data analysis; A. Herdean, conduction of experiments; T. Voss, conduction of experiments; D. Groelz, provision of study material or patients; A. Babayan, conduction of experiments; M. Tibbesma, conduction of experiments; M. Schlumpberger, provision of study material or patients; F. Chemi, conduction of experiments; D. Rothwell, conduction of experiments; H. Wikman, conduction of experiments; JP. Galizzi, conduction of experiments; I.R. Bergheim, conduction of experiments; H. Russnes, provision of study material or patients; B. Mussolin, conduction of experiments; S. Bonin, conduction of experiments; C. Voigt, conduction of experiments; $\mathrm{H}$. Musa, conduction of experiments; P. Pinzani, conduction of experi- 
ments; E. Lianidou, administrative support; K. Pantel, financial support, administrative support; M.R. Speicher, administrative support; C. Dive, administrative support; F. Betsou, provision of study material or patients; E. Schuuring, concept and study design, manuscript preparation; M. Kubista, provision of study material or patients; W. Ammerlaan, concept and study design, manuscript preparation; $M$. Sprenger-Haussels, concept and study design, manuscript preparation; T. Schlange, concept and study design, financial support, administrative support; E. Heitzer, concept and study design, data analysis, manuscript preparation.

Authors' Disclosures or Potential Conflicts of Interest: Upon manuscript submission, all authors completed the author disclosure form. Disclosures and/or potential conflicts of interest:

Employment or Leadership: R. Lampignano, Bayer AG; M.H.D. Neumann, Bayer AG, QIAGEN; V. Kloten, Lab GBU Research; A. Herdean, Tataa Biocenter; T. Voss, QIAGEN GmbH; D. Groelz, QIAGEN GmbH; M. Schlumpberger, QIAGEN GmbH; G. Brady, University of Manchester; K. Pantel, guest editor, Clinical Chemistry, AACC; M. Kubista, TATAA Biocenter AB; M. Sprenger-Haussels, QIAGEN GmbH; T. Schlange, Bayer AG.

Consultant or Advisory Role: T. Schlange, DeciBio.

Stock Ownership: D. Groelz, QIAGEN GmbH; M. Schlumpberger, QIAGEN; M. Kubista, TATAA Biocenter AB; M. Sprenger-Haussels, QIAGEN GmbH; T. Schlange, Bayer AG.

Honoraria: None declared.

Research Funding: The authors are participants in the Innovative Medicines Initiative consortium CANCER-ID. CANCER-ID is supported by the Innovative Medicines Initiative (IMI) Joint Undertaking under Grant Agreement no. 115749, resources of which are composed of financial contribution from the European Union's Seventh Framework Programme (FP7/2007-2013) and European Federation of Pharmaceutical Industries and Associations companies' in-kind contributions.

Several authors are participants in the European Union's Horizon 2020 SPIDIA4P project (Grant agreement no. 733112) or members of the Christian Doppler Research Laboratory for Liquid Biopsies for Early Detection of Cancer led by E. Heitzer funded by the Austrian Federal Ministry for Digital and Economic Affairs. H. Russnes, Health Region South East; M. Kubista, RVO: 86652036 and BIOCEV (CZ.1.05/ $1.1 .00 / 02.0109)$ from the ERDF.

Expert Testimony: None declared.

Patents: D. Groelz, WO2015140218 (A1); M. Sprenger-Haussels, WO2012EP68847, WO2012EP68892, WO2009EP03364, WO2013EP70016, WO2012EP68893.

Role of Sponsor: The funding organizations played no role in the design of study, choice of enrolled patients, review and interpretation of data, preparation of manuscript, or final approval of manuscript.

Acknowledgments: The authors thank Johanna Mielke (Bayer AG) for the support with statistical analysis. Centralized blood collection in Streck (Cell-Free DNA BCT®, Streck Inc., La Vista, USA) and PAXgene Blood ccfDNA Tube (PreAnalytiX GmbH, Hombrechtikon, Switzerland) was performed by the Integrated Biobank of Luxembourg (IBBL, Dudelange, Luxembourg, Informed Consent CNER No 201107/02 version 1.3 and subsequent amendments) and by QIAGEN (Hilden, Germany, Informed Consent approved by the ethic committee Ärztekammer Nordrhein), respectively.

\section{References}

1. Heitzer $E$, Ulz P, Geigl JB. Circulating tumor DNA as a liquid biopsy for cancer. Clin Chem 2015;61:112-23.

2. Leon SA, Shapiro B, Sklaroff DM, Yaros MJ. Free DNA in the serum of cancer patients and the effect of therapy. Cancer Res 1977;37:646-50.

3. Neumann MHD, Bender S, Krahn T, Schlange T. ctDNA and CTCs in liquid biopsy-current status and where we need to progress. Comput Struct Biotechnol J 2018;16:190-5.

4. Thierry AR, El Messaoudi S, Gahan PB, Anker P, Stroun M. Origins, structures, and functions of circulating DNA in oncology. Cancer Metastasis Rev 2016;35:347-76.

5. Heitzer E, Haque IS, Roberts CES, Speicher MR. Current and future perspectives of liquid biopsies in genomicsdriven oncology. Nat Rev Genet 2019;20:71.

6. Wan JCM, Massie C, Garcia-Corbacho J, Mouliere F, Brenton JD, Caldas C, et al. Liquid biopsies come of age: towards implementation of circulating tumour DNA. Nat Rev Cancer 2017;17:223-38.

7. Bronkhorst AJ, Aucamp J, Pretorius PJ. Cell-free DNA: preanalytical variables. Clin Chim Acta 2015; 450:243-53.

8. El Messaoudi S, Rolet F, Mouliere F, Thierry AR. Circulating cell free DNA: preanalytical considerations. Clin Chim Acta 2013;424:222-30.

9. Grölz D, Hauch S, Schlumpberger M, Guenther K, Voss T, Sprenger-Haussels $\mathrm{M}$, et al. Liquid biopsy preservation solutions for standardized pre-analytical workflowsvenous whole blood and plasma. Curr Pathobiol Rep 2018;6:275-86

10. Pantel K, Terstappen LW, Baggiani B, Krahn T, Schlange T. Abstract 1826: IMI's CANCER-ID: status of liquid biopsy standardization. Cancer Res 2016;76:1826.

11. Chan KCA, Yeung S-W, Lui W-B, Rainer TH, Lo YMD. Effects of preanalytical factors on the molecular size of cell-free DNA in blood. Clin Chem 2005:51:781-4

12. Chiu RWK, Poon LLM, Lau TK, Leung TN, Wong EMC, Lo YMD. Effects of blood-processing protocols on fetal and total DNA quantification in maternal plasma. Clin Chem 2001;47:1607-13.

13. Jung $M$, Klotzek $S$, Lewandowski $M$, Fleischhacker $M$, Jung K. Changes in concentration of DNA in serum and plasma during storage of blood samples. Clin Chem 2003;49:1028-9.

14. Parpart-Li S, Bartlett B, Popoli M, Adleff V, Tucker $L$, Steinberg $R$, et al. The effect of preservative and temperature on the analysis of circulating tumor DNA. Clin Cancer Res 2017;23:2471-7.

15. van Dessel LF, Beije N, Helmijr JCA, Vitale SR, Kraan J, Look MP, et al. Application of circulating tumor DNA in prospective clinical oncology trialsstandardization of preanalytical conditions. Mol Oncol 2017:11:295-304

16. Devonshire AS, Whale AS, Gutteridge A, Jones $G, C_{0}$ wen $S$, Foy CA, et al. Towards standardisation of cell-free DNA measurement in plasma: controls for extraction efficiency, fragment size bias and quantification. Anal Bioanal Chem 2014;406:6499-512.

17. Diefenbach RJ, Lee JH, Kefford RF, Rizos H. Evaluation of commercial kits for purification of circulating free DNA. Cancer Genet 2018;228-229:21-7.

18. Fleischhacker $M, S c h m i d t B$, Weickmann $S$, Fersching DMI, Leszinski GS, Siegele B, et al. Methods for isolation of cell-free plasma DNA strongly affect DNA yield. Clin Chim Acta 2011;412:2085-8.

19. Fong SL, Zhang JT, Lim CK, Eu KW, Liu Y. Comparison of 7 Methods for extracting cell-free DNA from serum samples of colorectal cancer patients. Clin Chem 2009;55:587-9.

20. Mauger F, Dulary C, Daviaud C, Deleuze J-F, Tost
J. Comprehensive evaluation of methods to isolate, quantify, and characterize circulating cell-free DNA from small volumes of plasma. Anal Bioanal Chem 2015;407:6873-8.

21. Page K, Guttery DS, Zahra N, Primrose L, Elshaw SR, Pringle $\mathrm{JH}$, et al. Influence of plasma processing on recovery and analysis of circulating nucleic acids. PLOS One 2013;8:10.

22. Pérez-Barrios $C$, Nieto-Alcolado I, Torrente $M_{\text {, }}$ Jiménez-Sánchez C, Calvo V, Gutierrez-Sanz L, et al. Comparison of methods for circulating cell-free DNA isolation using blood from cancer patients: impact on biomarker testing. Transl Lung Cancer Res 2016;5:665-72

23. Sorber L, Zwaenepoel K, Deschoolmeester V, Roeyen G, Lardon F, Rolfo C, et al. A comparison of cell-free DNA isolation kits: isolation and quantification of cell-free DNA in plasma. J Mol Diagn 2017;19:162-8.

24. van Dessel LF, Vitale SR, Helmijr JCA, Wilting SM, van der Vlugt-Daane M, Oomen-de Hoop E, et al. Highthroughput isolation of circulating tumor DNA: a comparison of automated platforms. Mol Oncol 2018;13:392-402.

25. Warton K, Graham L-J, Yuwono N, Samimi G. Comparison of 4 commercial kits for the extraction of circulating DNA from plasma. Cancer Genet 2018;228-229:143-50.

26. Broad Institute Cancer Cell Line Encyclopedia (CCLE). https://portals.broadinstitute.org/ccle (ACcessed January 2018).

27. Mohan S, Heitzer E, Ulz P, Lafer I, Lax S, Auer M, et al. Changes in colorectal carcinoma genomes under antiEGFR therapy identified by whole-genome plasma DNA sequencing. PLoS Genet 2014;10:3

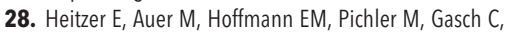


Ulz P, et al. Establishment of tumor-specific copy number alterations from plasma DNA of patients with cancer. Int J Cancer 2013;133:346-56

29. $R$ Core Team. $R$ : a language and environment for statistical computing 2013. http://www.R-project.org/ (ACcessed May 2014).

30. Cree IA, Uttley L, Buckley Woods H, Kikuchi H, Reiman $A, H a r n a n S$, et al. The evidence base for circulating tumour DNA blood-based biomarkers for the early detection of cancer: a systematic mapping review. BMC Cancer 2017;17:697.

31. Siravegna G, Marsoni S, Siena S, Bardelli A. Integrating liquid biopsies into the management of cancer. Nat Rev Clin Oncol 2017;14:531-48.

32. Kloten V, Rüchel N, Brüchle NO, Gasthaus J, Freudenmacher N, Steib F, et al. Liquid biopsy in colon cancer: comparison of different circulating DNA extraction systems following absolute quantification of KRAS muta- tions using Intplex allele-specific PCR. Oncotarget 2017;8:86253-63.

33. Sherwood JL, Corcoran C, Brown H, Sharpe AD, Musilova M, Kohlmann A. Optimised pre-analytical methods improve KRAS mutation detection in circulating tumour DNA (ctDNA) from patients with non-small cell lung cancer (NSCLC). PLoS One 2016;11:2.

34. Solassol J, Quantin $X$, Larrieux $M$, Senal R, Audran $P$, Grand $D$, et al. Comparison of five cell-free DNA isolation methods to detect the EGFR T790M mutation in plasma samples of patients with lung cancer. Clin Chem Lab Med 2018;56:e243-6.

35. Zhang R, Peng R, Li Z, Gao P, Jia S, Yang X, et al. Synthetic circulating cell-free DNA as quality control materials for somatic mutation detection in liquid biopsy for cancer. Clin Chem 2017;63:1465-75.

36. Jiang $P$, Chan CWM, Chan KCA, Cheng SH, Wong J, Wong VW-S, et al. Lengthening and shortening of plasma DNA in hepatocellular carcinoma patients. Proc Natl Acad Sci U S A 2015;112:E1317-25.

37. Mouliere F, Rosenfeld N. Circulating tumor-derived DNA is shorter than somatic DNA in plasma. Proc Natl Acad Sci U S A 2015;112:3178-9.

38. Mouliere F, Chandrananda D, Piskorz AM, Moore EK, Morris J, Ahlborn LB, et al. Enhanced detection of circulating tumor DNA by fragment size analysis. Sci Transl Med 2018;10: eaat4921.

39. Cristiano S, Leal A, Phallen J, Fiksel J, Adleff V, Bruhm DC, et al. Genome-wide cell-free DNA fragmentation in patients with cancer. Nature 2019;570:385-9.

40. Jahr S, Hentze H, Englisch S, Hardt D, Fackelmayer FO, Hesch R-D, et al. DNA fragments in the blood plasma of cancer patients: quantitations and evidence for their origin from apoptotic and necrotic cells. Cancer Res 2001;61:1659-65 